International Electronic Journal of Algebra

VOLUME 27 (2020) 271-287

DOI: $10.24330 /$ ieja.663094

\title{
HILBERT FUNCTIONS OF GRADED MODULES OVER AN EXTERIOR ALGEBRA: AN ALGORITHMIC APPROACH
}

\author{
Luca Amata and Marilena Crupi \\ Received: 8 July 2019; Revised: 10 October 2019; Accepted: 12 October 2019 \\ Communicated by Alberto Tonolo
}

\begin{abstract}
Let $K$ be a field, $E$ the exterior algebra of a finite dimensional $K$-vector space, and $F$ a finitely generated graded free $E$-module with homogeneous basis $g_{1}, \ldots, g_{r}$ such that $\operatorname{deg} g_{1} \leq \operatorname{deg} g_{2} \leq \cdots \leq \operatorname{deg} g_{r}$. Given the Hilbert function of a graded $E$-module of the type $F / M$, with $M$ graded submodule of $F$, the existence of the unique lexicographic submodule of $F$ with the same Hilbert function as $M$ is proved by a new algorithmic approach. Such an approach allows us to establish a criterion for determining if a sequence of nonnegative integers defines the Hilbert function of a quotient of a free $E$ module only via the combinatorial Kruskal-Katona's theorem.
\end{abstract}

Mathematics Subject Classification (2010): 13A02, 15A75, 18G10, 68W30 Keywords: Exterior algebra, Hilbert function, monomial submodule, lexicographic submodule

\section{Introduction}

The notion of Hilbert function is central in commutative algebra and in algebraic geometry and is important in combinatorics and in computational algebra. Let $K$ be a field and $A$ a graded $K$-algebra. The Hilbert function of a graded $K$-algebra $A$ computes the vector space dimension of its graded components. It encodes important information on $A$ such as its Krull dimension or its multiplicity [11]. The possible Hilbert functions of standard graded $K$-algebra $A$ are described by Macaulay's theorem $[14,15]$. The Hilbert function of the Stanley-Reisner ring of a simplicial complex $\Delta$ is determined by the $f$-vector of $\Delta$, and vice versa. The possible $f$-vectors of a simplicial complex are characterized in the theorem of Kruskal-Katona $[12,13]$. This theorem is the "squarefree" analogue of Macaulay's theorem and may be also interpreted as a theorem on Hilbert functions of quotients of exterior algebras in [5]. 
Let $K$ be a field, $V$ a $K$-vector space with basis $e_{1}, \ldots, e_{n}$, and $E$ the exterior algebra of $V$. Let $F=\oplus_{i=1}^{r} E g_{i}$ be a finitely generated graded free $E$-module with homogeneous basis $g_{1}, \ldots, g_{r}$ such that $\operatorname{deg} g_{1} \leq \operatorname{deg} g_{2} \leq \cdots \leq \operatorname{deg} g_{r}$.

In [4], the authors of this paper have given a generalization of the combinatorial Kruskal-Katona theorem [5, Theorem 4.1] for finitely generated modules over exterior algebras. More precisely, they have described the possible Hilbert functions of graded $E$-modules of the form $F / M$, with $M$ graded submodule of the free $E$-module $F$ via the class of lexicographic submodules (see also [3]). The construction of such a submodule has been realized using the classical way (which involves suitable sets of monomials of $F$ ). More in details, if $M$ is a graded submodule of $F$, the construction of the lexicographic submodule $M^{\text {lex }}$ with the same Hilbert function of $M$ proceeds as follows: for each graded component $M_{j}$ of $M$, let $M_{j}^{\text {lex }}$ be the $K$-vector space spanned by the (unique) lexicographic segment $L_{j}$ of $F$ (Definition 2.5) with $\left|L_{j}\right|=\operatorname{dim}_{K} M_{j}$. Then one defines $M^{\text {lex }}=\oplus_{j} M_{j}^{\text {lex }}$. However, the calculations could be tedious. Hereafter, we describe an alternative way for determining the lexicographic submodule we are looking for. Our approach (Theorem 3.2) manipulates sequences of nonnegative integers. More in details, if $M$ is a graded submodule of $F$, we associate to $F / M$ the sequence $H s_{F / M}=\left(H_{F / M}\left(f_{1}\right), H_{F / M}\left(f_{1}+1\right), \ldots, H_{F / M}\left(f_{r}+n\right)\right) \in \mathbb{N}_{0}^{f_{r}+n-f_{1}+1}$. We call $H s_{F / M}$ the Hilbert sequence of the graded $E$-module $F / M$. Using the KruskalKatona theorem (Theorem 2.9), and operating on the given Hilbert sequence by repeated subtractions, one obtains $r$ suitable sequences which are the Hilbert sequences of $r$ graded $K$-algebras $E / I_{i}$, with $I_{i}(i=1, \ldots, r)$ lexicographic ideals of $E$, and $L=\oplus_{i=1}^{r} I_{i} g_{i}$ will be the unique lexicographic submodule of $F$ with $H_{F / L}=H_{F / M}$. Consequently, we get a new criterion (Criterion 3.3) that, given a sequence $H$ of nonnegative integers (of a certain length), finds out if $H$ determines the Hilbert function of a quotient of the type $F / M$.

The paper is organized as follows. Section 2 contains preliminary notions and results. Moreover, we discuss in details the Hilbert functions of graded $E$-modules. In Section 3, we describe a new procedure for the construction of the unique lexicographic submodule for a given Hilbert function (Theorem 3.2). Furthermore, a new criterion (Criterion 3.3) to verify if a sequence of nonnegative integers determines the Hilbert function of quotients of graded $E$-modules is given. Finally, Section 4 contains some examples illustrating our procedures.

All the examples in the paper are constructed by a Macaulay2 package created by the authors of this article. 


\section{Preliminaries and notations}

Let $K$ be a field. We denote by $E=K\left\langle e_{1}, \ldots, e_{n}\right\rangle$ the exterior algebra of a $K$ vector space $V$ with basis $e_{1}, \ldots, e_{n}$. For any subset $\sigma=\left\{i_{1}, \ldots, i_{d}\right\}$ of $\{1, \ldots, n\}$ with $i_{1}<i_{2}<\cdots<i_{d}$ we write $e_{\sigma}=e_{i_{1}} \wedge \ldots \wedge e_{i_{d}}$, and call $e_{\sigma}$ a monomial of degree $d$. We set $e_{\sigma}=1$, if $\sigma=\emptyset$. The set of monomials in $E$ forms a $K$-basis of $E$ of cardinality $2^{n}$.

In order to simplify the notation, we put $f g=f \wedge g$ for any two elements $f$ and $g$ in $E$. An element $f \in E$ is called homogeneous of degree $j$ if $f \in E_{j}$, where $E_{j}=\bigwedge^{j} V$. An ideal $I$ is called graded if $I$ is generated by homogeneous elements. If $I$ is graded, then $I=\oplus_{j \geq 0} I_{j}$, where $I_{j}$ is the $K$-vector space of all homogeneous elements $f \in I$ of degree $j$. We denote by $\operatorname{indeg}(I)$ the initial degree of $I$, that is, the minimum $s$ such that $I_{s} \neq 0$.

Let $\mathcal{M}$ be the category of finitely generated $\mathbb{Z}$-graded left and right $E$-modules $M$ satisfying $a m=(-1)^{\operatorname{deg} a \operatorname{deg} m} m a$ for all homogeneous elements $a \in E, m \in M$.

If $M \in \mathcal{M}$, the function $H_{M}: \mathbb{Z} \rightarrow \mathbb{Z}$ given by $H_{M}(d)=\operatorname{dim}_{K} M_{d}$ is called the Hilbert function of $M[6,9]$.

For any not empty subset $S$ of $E$ (respectively, of $F$ ), we denote by $\operatorname{Mon}(S)$ the set of all monomials in $S$ (respectively, of $F$ ), and we denote its cardinality by $|S|$. Moreover, we denote by $\operatorname{Mon}_{d}(S)$ the set of all monomials of degree $d$ in $S$.

Let $F \in \mathcal{M}$ be a free module with homogeneous basis $g_{1}, \ldots, g_{r}$, where $\operatorname{deg}\left(g_{i}\right)=$ $f_{i}$ for each $i=1, \ldots, r$, with $f_{1} \leq f_{2} \leq \cdots \leq f_{r}$. We write $F=\oplus_{i=1}^{r} E g_{i}$. The elements of the form $e_{\sigma} g_{i}$, where $e_{\sigma} \in \operatorname{Mon}(E)$, are called monomials of $F$, and $\operatorname{deg}\left(e_{\sigma} g_{i}\right)=\operatorname{deg}\left(e_{\sigma}\right)+\operatorname{deg}\left(g_{i}\right)$.

Definition 2.1. A graded submodule $M$ of $F$ is a monomial submodule if $M$ is a submodule generated by monomials of $F$, i.e.,

$$
M=I_{1} g_{1} \oplus \cdots \oplus I_{r} g_{r}
$$

with $I_{i}$ a monomial ideal of $E$, for each $i$.

In the study of the behavior of the Hilbert function of a graded E-module, the class of lexicographic modules plays a fundamental role.

Denote by $>_{\text {lex }}$ the lexicographic order (lex order, for short) on $\operatorname{Mon}_{d}(E)$, i.e., if $e_{\sigma}=e_{i_{1}} e_{i_{2}} \cdots e_{i_{d}}$ and $e_{\tau}=e_{j_{1}} e_{j_{2}} \cdots e_{j_{d}}$ are monomials belonging to $\operatorname{Mon}_{d}(E)$ with $1 \leq i_{1}<i_{2}<\cdots<i_{d} \leq n$ and $1 \leq j_{1}<j_{2}<\cdots<j_{d} \leq n$, then $e_{\sigma}>_{\operatorname{lex}} e_{\tau}$ if $i_{1}=j_{1}, \ldots, i_{s-1}=j_{s-1}$ and $i_{s}<j_{s}$ for some $1 \leq s \leq d$. 
Definition 2.2. A nonempty subset $M$ of $\operatorname{Mon}_{d}(E)$ is called a lexicographic segment (lex segment, for short) of degree $d$ if for all $v \in M$ and all $u \in \operatorname{Mon}_{d}(E)$ such that $u>_{\text {lex }} v$, we have that $u \in M$.

Definition 2.3. A monomial ideal $I$ of $E$ is called a lexicographic ideal (lex ideal, for short) if for all monomials $v \in I$ and all monomials $u \in E$ with $\operatorname{deg} v=\operatorname{deg} u$ and $u>_{\text {lex }} v$, then $u \in I$, i.e., $\operatorname{Mon}_{d}(I)$ is a lex segment, for all $d$.

Remark 2.4. The trivial ideals of $E$ are monomial lex ideals.

It is well-known that if $I$ is a graded ideal of $E$, then there exists a unique lex segment ideal of $E$, usually denoted by $I^{\text {lex }}$, such that $H_{E / I}=H_{E / I^{\text {lex }}}[5$, Theorem 4.1] (see also [11, Theorem 6.3.1]).

Now, let $F_{d}$ be the part of degree $d$ of $F=\oplus_{i=1}^{r} E g_{i}$, i.e., the $K$-vector space of homogeneous elements of $F$ of degree $d$. Denote by $\operatorname{Mon}_{d}(F)$ the set of all monomials of degree $d$ of $F$. We order such a set by the ordering $>_{\operatorname{lex}_{F}}$ defined as follows:

if $u g_{i}$ and $v g_{j}$ are monomials of $F$ such that $\operatorname{deg}\left(u g_{i}\right)=\operatorname{deg}\left(v g_{j}\right)$, then $u g_{i}>_{\operatorname{lex}_{F}} v g_{j}$ if $i<j$ or $i=j$ and $u>_{\operatorname{lex}} v$.

For instance, if $E=K\left\langle e_{1}, e_{2}, e_{3}\right\rangle$ and $F=E g_{1} \oplus E g_{2}$, with $\operatorname{deg} g_{1}=2$ and $\operatorname{deg} g_{2}=3$, the monomials of $F$ of degree 4 , with respect to $>>_{\operatorname{lex}_{F}}$, are ordered as follows:

$$
e_{1} e_{2} g_{1}>_{\operatorname{lex}_{F}} e_{1} e_{3} g_{1}>_{\operatorname{lex}_{F}} e_{2} e_{3} g_{1}>_{\operatorname{lex}_{F}} e_{1} g_{2}>_{\operatorname{lex}_{F}} e_{2} g_{2}>_{\operatorname{lex}_{F}} e_{3} g_{2} .
$$

Definition 2.5. A nonempty subset $N$ of $\operatorname{Mon}_{d}(F)$ is called a lexicographic segment of $F$ (lex $F$ segment, for short) of degree $d$ if for all $v \in N$ and all $u \in \operatorname{Mon}_{d}(F)$ such that $u>\operatorname{lex}_{F} v$, then $u \in N$.

Definition 2.6. Let $L$ be a monomial submodule of $F$. $L$ is a lexicographic submodule (lex submodule, for short) if for all $u, v \in \operatorname{Mon}_{d}(F)$ with $v \in L$ and $u>_{\operatorname{lex}_{F}} v$, one has $u \in L$, for every $d$, i.e., $\operatorname{Mon}_{d}(L)$ is a $\operatorname{lex}_{F}$ segment of degree $d$, for each degree $d$.

Definition 2.6 is equivalent to the following one [3, Proposition 3.12] (see also [7, Proposition 3.8]).

Definition 2.7. Let $L$ be a graded submodule of $F$. $L$ is a lex submodule of $F$ if $L=\oplus_{i=1}^{r} I_{i} g_{i}$, with $I_{i}$ lex ideals of $E(i=1, \ldots, r)$, and $\left(e_{1}, \ldots, e_{n}\right)^{\rho_{i}+f_{i}-f_{i-1}} \subseteq$ $I_{i-1}$, for $i=2, \ldots, r$, with $\rho_{i}=\operatorname{indeg} I_{i}$. 
Example 2.8. Let $E=K\left\langle e_{1}, e_{2}, e_{3}, e_{4}\right\rangle$ and $F=E g_{1} \oplus E g_{2} \oplus E g_{3}$, with $\operatorname{deg} g_{1}=$ $-2, \operatorname{deg} g_{2}=-1$ and $\operatorname{deg} g_{3}=3$. The submodule

$$
L=\left(e_{1} e_{2}, e_{1} e_{3}, e_{2} e_{3} e_{4}\right) g_{1} \oplus\left(e_{1} e_{2}, e_{1} e_{3}, e_{1} e_{4}, e_{2} e_{3}\right) g_{2} \oplus\left(e_{1} e_{2} e_{3}, e_{1} e_{2} e_{4}\right) g_{3}
$$

is a lex submodule of $F$.

In order to keep the paper self-contained, we recall some notions from [4].

If $a=\left(a_{1}, \ldots, a_{p}\right)$ and $b=\left(b_{1}, \ldots, b_{p}\right)$ are two sequences of nonnegative integers, we say that $a>b$ if $\left(a_{1}, \ldots, a_{p}\right)>\left(b_{1}, \ldots, b_{p}\right)$ in the lexicographic ordering, i.e., the difference $a_{s}-b_{s}$ is positive for the first index $1 \leq s \leq p$ where it is not zero.

We make the following conventions:

$$
\left(\begin{array}{c}
m \\
k
\end{array}\right)=0 \quad \text { if } \quad m<k \quad \text { or } \quad k<0 .
$$

One can observe that if $F=\oplus_{i=1}^{r} E g_{i}$, we have that

$$
H_{F}(d)=\sum_{i=1}^{r} H_{E g_{i}}(d)=\sum_{i=1}^{r}\left(\begin{array}{c}
n \\
d-f_{i}
\end{array}\right),
$$

and consequently, if $M$ is a graded submodule of $F$, one has

$$
H_{F / M}(d)+H_{M}(d)=\sum_{i=1}^{r}\left(\begin{array}{c}
n \\
d-f_{i}
\end{array}\right)
$$

where $\left(\begin{array}{c}n \\ d-f_{i}\end{array}\right)$ is the number of monomials of degree $d-f_{i}$ in $F$.

Let $a$ and $i$ be two positive integers. Then $a$ has the unique $i$-th Macaulay expansion [11, Lemma 6.3.4]

$$
a=\left(\begin{array}{c}
a_{i} \\
i
\end{array}\right)+\left(\begin{array}{c}
a_{i-1} \\
i-1
\end{array}\right)+\cdots+\left(\begin{array}{c}
a_{j} \\
j
\end{array}\right)
$$

with $a_{i}>a_{i-1}>\cdots>a_{j} \geq j \geq 1$. We define

$$
a^{(i)}=\left(\begin{array}{c}
a_{i} \\
i+1
\end{array}\right)+\left(\begin{array}{c}
a_{i-1} \\
i
\end{array}\right)+\cdots+\left(\begin{array}{c}
a_{j} \\
j+1
\end{array}\right) .
$$

We also set $0^{(i)}=0$ for all $i \geq 1$.

The next result, known as the Kruskal-Katona theorem, classifies Hilbert functions of quotients of exterior algebras.

Theorem 2.9. ([5, Theorem 4.1]) Let $\left(h_{1}, \ldots, h_{n}\right)$ be a sequence of nonnegative integers. Then the following conditions are equivalent:

(a) $1+\sum_{i=1}^{n} h_{i} t^{i}$ is the Hilbert series of a graded $K$-algebra $E / I$;

(b) $0<h_{i+1} \leq h_{i}^{(i)}, 0<i \leq n-1$. 
From now on, if $1+\sum_{i=1}^{n} h_{i} t^{i}$ is the Hilbert series of a graded $K$-algebra $E / I$, $I \subsetneq E$, the sequence $\left(1, h_{1}, \ldots, h_{n}\right)$ is called the Hilbert sequence of $E / I$. We will denote it by $H s_{E / I}$.

From the Kruskal-Katona theorem, one can deduce that a sequence of nonnegative integers $\left(h_{0}, h_{1}, \ldots, h_{n}\right)$ is the Hilbert sequence of a graded $K$-algebra $E / I$, with $I \subsetneq E$ graded ideal of initial degree $\geq 1$, if $h_{0}=1, h_{1} \leq n$ and condition (b) in Theorem 2.9 holds. Note that if $I=0$, then $H s_{E / I}=H s_{E}=\left(1, n,\left(\begin{array}{l}n \\ 2\end{array}\right), \cdots,\left(\begin{array}{l}n \\ n\end{array}\right)\right)$.

Finally, we set $H s_{E / I}=(\underbrace{0, \ldots, 0}_{n+1})$, if $I=E$.

Let us consider the graded $E$-module $F=\oplus_{i=1}^{r} E g_{i}$. One can quickly verify that

$$
H_{F}(d)=\operatorname{dim}_{K} F_{d}=0, \quad \text { for } \quad d<f_{1} \quad \text { and } \quad d>f_{r}+n .
$$

Now, we discuss the Hilbert function of a graded $E$-algebra $F / M$, with $M$ submodule of $F$.

Discussion 2.10. Assume $M$ is a monomial submodule of $F$. From (1), it follows that

$$
H_{F / M}(t)=\sum_{i=f_{1}}^{f_{r}+n} H_{F / M}(i) t^{i},
$$

and we can associate to $F / M$ the following sequence

$$
\left(H_{F / M}\left(f_{1}\right), H_{F / M}\left(f_{1}+1\right), \ldots, H_{F / M}\left(f_{r}+n\right)\right) \in \mathbb{N}_{0}^{f_{r}+n-f_{1}+1} .
$$

Such a sequence is called the Hilbert sequence of $F / M$ and it is denoted by $H s_{F / M}$. The integers $f_{1}, f_{1}+1, \ldots, f_{r}+n$ are called the $H s_{F / M}$-degrees. It is clear that $H s_{F / M} \leq H s_{F}$.

Moreover, we define

$$
\operatorname{indeg} H s_{F / M}=\min \left\{d: H_{F / M}(d) \neq 0\right\}, \quad \text { for } d=f_{1}, \ldots, f_{r}+n .
$$

We use the standard notation $[p]$ for the set $\{1,2, \ldots, p\}$.

Consider the sequence $H s_{F / M}$ defined in (2). The entries $H_{F / M}\left(f_{i}\right)(i=1, \ldots, r)$ are called the critical values of $H s_{F / M}$. Moreover, we define

$$
\mu_{f_{i}}=\left|\left\{s \in[r]: f_{s}=f_{i}\right\}\right|, \quad \text { for } i=1,2, \ldots, r,
$$

and we call $\mu_{f_{i}}$ the multiplicity of $H_{F / M}\left(f_{i}\right)$.

Now, let us consider the case $H_{F / M}\left(f_{1}\right)=0$. In such a situation, one has:

$$
M=E g_{1} \oplus T_{2},
$$

where $T_{2}$ is a submodule of $E g_{2} \oplus \cdots \oplus E g_{r}$. Indeed, if $H_{F / M}\left(f_{1}\right)=0$, then $M_{f_{1}}=F_{f_{1}}$ and so $M_{j}=F_{j}$, for $j=f_{1}, \ldots, f_{2}-1$. Hence, $H_{F / M}(j)=0$, for $j=f_{1}, \ldots, f_{2}-1$. 
Now, let us consider the critical value $H_{F / M}\left(f_{2}\right)$.

If $H_{F / M}\left(f_{2}\right)=0$, we can repeat the same reasoning done for $H_{F / M}\left(f_{1}\right)=0$, i.e., $H_{F / M}(j)=0$, for $j=f_{2}, \ldots, f_{3}-1$, and $M=E g_{1} \oplus E g_{2} \oplus T_{3}$, where $T_{3}$ is a submodule of $E g_{3} \oplus \cdots \oplus E g_{r}$. And so on.

Now, let $k$ be the minimum integer such that $H_{F / M}\left(f_{k}\right) \neq 0$, i.e., indeg $H s_{F / M}=$ $f_{k}$. Note that $M=E g_{1} \oplus \cdots \oplus E g_{k-1} \oplus T_{k}$, where $T_{k}$ is a submodule of $E g_{k} \oplus$ $\cdots \oplus E g_{r}$. We have:

$$
H_{F / M}\left(f_{k}\right) \leq \mu_{f_{k}},
$$

and

$$
H_{F / M}\left(f_{k}+1\right) \leq n \mu_{f_{k}}+\mu_{f_{k}+1} .
$$

The integer $H_{F / M}\left(f_{k}\right)$ is called the initial critical value (of $F / M$ ) and $f_{k}$ the initial critical degree (of $F / M$ ).

A classification of Hilbert functions of quotients of graded free $E$-modules can be found in [4]. The key point of such a classification is that if $M$ is a graded submodule of $F$, then there exists a unique lex submodule of $F$ with the same Hilbert function as $M$ denoted by $M^{\text {lex }}$.

\section{The Lex-Algorithm}

In this Section, fixed a graded submodule $M$ of $F$, we give a new procedure for the construction of $M^{\text {lex }}$. The algorithmic construction of the lex submodule is based on the additive property of Hilbert functions and on Kruskal-Katona's theorem. The idea dates back to the computation of all admissible Hilbert sequences of quotients of exterior algebras in [2] and from the realisation that, given a Hilbert sequence $H s_{F / M}$, there exist only $r=\operatorname{rank} F$ Hilbert sequences of the type $E / I$ ( $I$ graded ideal in $E$ ), which determine $M^{\text {lex }}$. The choice of such $r$ sequence is forced by some restrictions, as next theorem will point out.

Let $p, q \in \mathbb{Z}$ such that $p<q$. A finite sequence $H$ of nonnegative integers is called $[p, q]$-sequence if it is indexed by the set $[p, q]$ :

$$
H=\left(h_{i}\right)_{i \in[p, q]}=\left(h_{p}, h_{p+1}, \ldots, h_{q}\right) .
$$

We set

$$
H(j)=h_{j}, \quad \text { for } \quad j \in[p, q]
$$

the integers $j$ are called $H$-degrees.

One can observe that the sequence $H s_{F / M}$ is a $\left[f_{1}, f_{r}+n\right]$-sequence, and the integers $j \in\left[f_{1}, f_{r}+n\right]$ are the $H s_{F / M}$-degrees.

Moreover, if $p=0$, then $H$ is the $(q+1)$-tuple $\left(h_{0}, h_{1}, \ldots, h_{q}\right)$. 
Example 3.1. Let $p=-2$ and $q=1$. Then $[-2,1]=\{-2,-1,0,1\}$. If $H=$ $(0,2,7,3)$ is a $[-2,1]$-sequence, one has $H(-2)=0, H(-1)=2, H(0)=7$, and $H(1)=3$.

Theorem 3.2. (The Lex-Algorithm) Let $\left(h_{f_{1}}, \ldots, h_{f_{r}+n}\right)$ be the Hilbert sequence of a graded $E$ - module $F / M$. Then, there exists a unique lex submodule $L$ of $F$ such that $H_{F / L}=H_{F / M}$.

Proof. Set $H s_{F / M}=\left(h_{f_{1}}, \ldots, h_{f_{r}+n}\right)$. We want to construct a lex submodule $L=\oplus_{i=1}^{r} I_{i} g_{i}$ of $F$ such that $H_{F / L}=H_{F / M}$. Let us define

$$
0_{p}=(0, \ldots, 0) \in \mathbb{N}^{p}, \text { for } p \geq 1
$$

Step 1. Construction of $I_{r}$.

Let us consider the following subsequence of $H s_{F / M}$ :

$$
\left(h_{f_{r}}, \ldots, h_{f_{r}+n}\right)=\left(H_{F / M}\left(f_{r}\right), \ldots, H_{F / M}\left(f_{r}+n\right)\right) .
$$

Define

- $H_{r}(0):=\min \left\{1, H_{F / M}\left(f_{r}\right)\right\}$

- $H_{r}(1):= \begin{cases}\min \left\{n, H_{F / M}\left(f_{r}+1\right)\right\} & \text { if } H_{r}(0)=1 \\ 0 & \text { if } H_{r}(0)=0,\end{cases}$

- $H_{r}(2):=\min \left\{H_{r}(1)^{(1)}, H_{F / M}\left(f_{r}+2\right)\right\}$,

- $H_{r}(i):=\min \left\{H_{r}(i-1)^{(i-1)}, H_{F / M}\left(f_{r}+i\right)\right\}$, for $3 \leq i \leq n$.

Setting $H_{r}=\left(H_{r}(0), \ldots, H_{r}(n)\right)$, if $H_{r}(0)=1$, then Kruskal-Katona's theorem (Theorem 2.9) assures that such a sequence is the largest extractable Hilbert sequence from (3) for which there exists a lex ideal $I_{r} \subsetneq E$ such that

$$
H s_{E / I_{r}}=H_{r}
$$

on the contrary, if $H_{r}(0)=0$, then the only admissible Hilbert sequence is the null sequence. In such a case, the corresponding lex ideal is $I_{r}=E$.

Step 2. Construction of $I_{r-1}$.

Let us define

$$
\widetilde{H}_{r}=0_{f_{r}-f_{1}} \uplus H s_{E / I_{r}}=(\underbrace{0, \ldots, 0}_{f_{r}-f_{1}}, H_{r}(0), \ldots, H_{r}(n)),
$$

and consider the $\left[f_{1}, f_{r}+n\right]$-sequence

$$
\begin{aligned}
H s_{F / M}-\widetilde{H}_{r} & =\left(h_{f_{1}}, \ldots, h_{f_{r}-1}, h_{f_{r}}-H_{r}(0), \ldots, h_{f_{r}+n}-H_{r}(n)\right)= \\
& =(h_{f_{1}}, \ldots, h_{f_{r}-1}, h_{f_{r}}-H_{r}(0), \ldots, h_{f_{r-1}+n}-H_{r}\left(n-f_{r}+f_{r-1}\right), \underbrace{0, \ldots, 0}_{f_{r}-f_{r-1}}) .
\end{aligned}
$$


Note that if $f_{r-1}<f_{r}$, then the last $f_{r}-f_{r-1}$ entries of $H s_{F / M}$ concern only the ideal $I_{r}$. Furthermore, if $f_{1}=f_{2}=\ldots=f_{r}$, then $\widetilde{H}_{r}=H_{r}$.

Set

$$
\bar{H}_{r}=H s_{F / M}-\widetilde{H}_{r} .
$$

Starting from the $(r-1)$-th critical degree, we can repeat on $\bar{H}_{r}$ the same reasoning done for $H s_{F / M}$. More precisely, define

$$
\begin{aligned}
& \text { - } H_{r-1}(0):=\min \left\{1, \bar{H}_{r}\left(f_{r-1}\right)\right\}, \\
& \text { - } H_{r-1}(1):= \begin{cases}\min \left\{n, \bar{H}_{r}\left(f_{r-1}+1\right)\right\} & \text { if } H_{r-1}(0)=1 \\
0 & \text { if } H_{r-1}(0)=0,\end{cases} \\
& \text { - } H_{r-1}(2):=\min \left\{H_{r-1}(1)^{(1)}, \bar{H}_{r}\left(f_{r-1}+2\right)\right\}, \\
& \text { - } H_{r-1}(i):=\min \left\{H_{r-1}(i-1)^{(i-1)}, \bar{H}_{r}\left(f_{r-1}+i\right)\right\}, \text { for } 3 \leq i \leq n,
\end{aligned}
$$

and let $I_{r-1}$ be the unique lex ideal of $E$ such that

$$
H s_{E / I_{r-1}}=H_{r-1}=\left(H_{r-1}(0), \ldots, H_{r-1}(n)\right) .
$$

Setting

$$
\widetilde{H}_{r-1}=0_{f_{r-1}-f_{1}} \uplus H s_{E / I_{r-1}} \uplus 0_{f_{r}-f_{r-1}}=(\underbrace{0, \ldots, 0}_{f_{r-1}-f_{1}}, H_{r-1}(0), \ldots, H_{r-1}(n), \underbrace{0, \ldots, 0}_{f_{r}-f_{r-1}}),
$$

let us consider the $\left[f_{1}, f_{r}+n\right]$-sequence

$$
\begin{aligned}
& \bar{H}_{r-1}=\bar{H}_{r}-\widetilde{H}_{r-1}=\left(\bar{H}_{r}\left(f_{1}\right), \ldots, \bar{H}_{r}\left(f_{r-1}-1\right), \bar{H}_{r}\left(f_{r-1}\right)-H_{r-1}(0), \ldots\right. \\
& \ldots, \bar{H}_{r}\left(f_{r-1}+n\right)-H_{r-1}(n), \underbrace{0, \ldots, 0}_{f_{r}-f_{r-1}})= \\
& =\left(h_{f_{1}}, \ldots, h_{f_{r-1}-1}, \bar{H}_{r}\left(f_{r-1}\right)-H_{r-1}(0), \ldots, \bar{H}_{r}\left(f_{r-2}+n\right)-H_{r-1}\left(n-f_{r-1}+f_{r-2}\right),\right. \\
& \underbrace{0, \ldots, 0}_{f_{r}-f_{r-2}}) \text {. }
\end{aligned}
$$

Proceeding as before, we will get a Hilbert sequence $H_{r-2}$ and a lex ideal $I_{r-2}$ such that $H_{r-2}=H s_{E / I_{r-2}}$. Finally, iterating the previous procedure, after $r$ steps, we will obtain $r$ lex ideals $I_{r}, \ldots, I_{1}$. The monomial submodule $L=\oplus_{i=1}^{r} I_{i} g_{i}$ is the lex submodule we are looking for. Indeed, the suitable choice of the $r$ Hilbert sequences $H_{r}, H_{r-1}, \ldots, H_{1}$ assures that $L_{d}$ is generated (as a $K$-vector space) by a $\operatorname{lex}_{F}$ segment of monomials of degree $d$ of $F$.

Note that the $r$ subtractions will return the $\left(f_{r}+n-f_{1}+1\right)$-tuple $0_{f_{r}+n-f_{1}+1}$, and consequently $H_{F / M}=H_{F / L}$, i.e., $H s_{F / M}=H s_{F / L}=\sum_{i=1}^{r} \widetilde{H}_{i}$.

In order to outline the basic idea behind the Theorem 3.2, we present a sketch of the algorithm as pseudocode in Figure 1. 


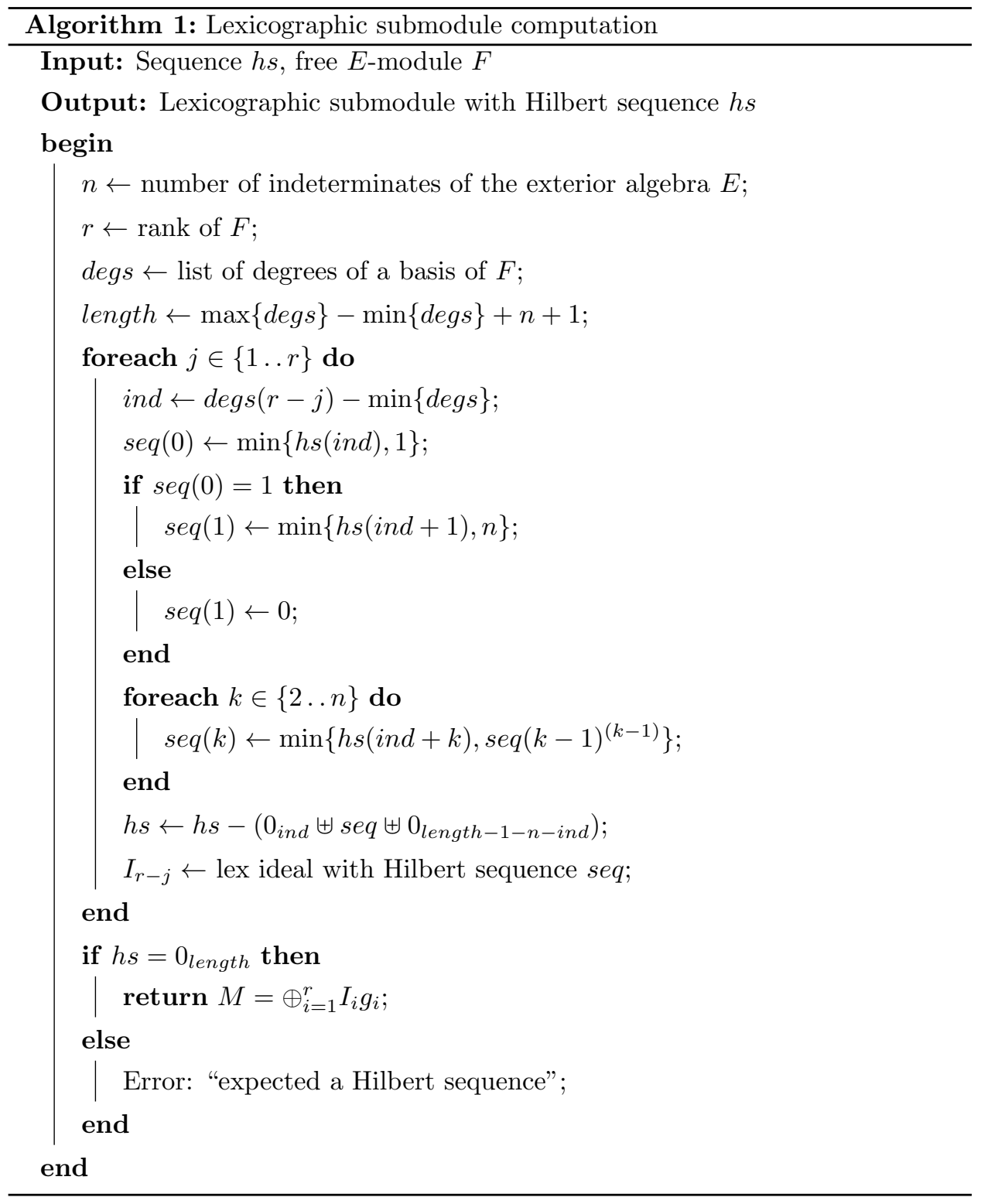

Figure 1. Algorithm in Theorem 3.2

The procedure in Theorem 3.2, allows us to give a criterion for determining when a sequence of nonnegative integers is the Hilbert function of a graded $E$-algebra of the type $F / M$, with $M$ graded submodule of $F$.

Criterion 3.3. Let $F=\oplus_{i=1}^{r} E g_{i}$ be a finitely generated graded free $E$-module and the generators $g_{i}$ of degrees $f_{i}$ are ordered such that $f_{1} \leq f_{2} \leq \cdots \leq f_{r}$. 
A sequence of nonnegative integers

$$
H=\left(h_{f_{1}}, \ldots, h_{f_{r}+n}\right)
$$

is the Hilbert sequence of graded $E$-module $F / M$, if applying the algorithm in Theorem 3.2, after $r$ steps, the repeated subtractions from $H$ of the largest Hilbert sequences (in the sense of the aforementioned theorem) of graded $K$-algebras of the type $E / I$, return the null sequence $0_{f_{r}+n-f_{1}+1}$.

\section{Examples}

In this Section, we collect some examples in order to illustrate the strategy used in Theorem 3.2.

In order to simplify the notation, once we fix a sequence of nonnegative integers $H$, when we say that a graded ideal $I$ of $E$ has $H$ as Hilbert sequence, or that $H$ is the Hilbert sequence of a graded ideal $I$, we mean that $H s_{E / I}=H$. Moreover, in what follows, we refer to Hilbert sequences of quotients of the type $E / I$ ( $I$ graded ideal of $E$ ), whenever it is not specified.

Example 4.1. Let $E=K\left\langle e_{1}, e_{2}, e_{3}, e_{4}\right\rangle, F=E^{3}$ and

$$
M=\left(e_{1} e_{2}, e_{3} e_{4}\right) g_{1} \oplus\left(e_{1} e_{2}, e_{2} e_{3} e_{4}\right) g_{2} \oplus\left(e_{2} e_{3} e_{4}\right) g_{3}
$$

a submodule of $F$. $M$ is not a lex submodule of $F$. The Hilbert sequence of $F / M$ is

$$
H s_{F / M}=(3,12,15,4,0) .
$$

Setting $J_{1}=\left(e_{1} e_{2}, e_{3} e_{4}\right), J_{2}=\left(e_{1} e_{2}, e_{2} e_{3} e_{4}\right)$ and $J_{3}=\left(e_{2} e_{3} e_{4}\right)$, one has

$$
H s_{E / J_{1}}=(1,4,4,0,0), H s_{E / J_{2}}=(1,4,5,1,0), H s_{E / J_{3}}=(1,4,6,3,0),
$$

and $H_{F / M}(d)=\sum_{i=1}^{3} H_{E / J_{i}}(d), d \geq 0$, as the next table shows

\begin{tabular}{|l|ccccc|c|}
\hline Hs-degrees & $\mathbf{0}$ & 1 & 2 & 3 & 4 & \\
\hline$H s_{E / J_{1}}$ & $(\mathbf{1}$, & $\mathbf{4}$ & $\mathbf{4}$ & $\mathbf{0}$, & $\mathbf{0})$ & + \\
$H s_{E / J_{2}}$ & $(\mathbf{1}$, & $\mathbf{4}$, & $\mathbf{5}$, & $\mathbf{1}$, & $\mathbf{0})$ & + \\
$H s_{E / J_{3}}$ & $(\mathbf{1}$, & $\mathbf{4}$, & $\mathbf{6}$, & $\mathbf{3}$, & $\mathbf{0})$ & $=$ \\
\hline$H s_{F / M}$ & $(3$, & 12, & 15, & 4, & $0)$ & \\
\cline { 1 - 3 } & & &
\end{tabular}

Now, we want to describe our new point of view.

Let us consider the sequence $H=(3,12,15,4,0)$. The largest Hilbert sequence of a graded ideal that can be extracted from $H$ is $H_{3}=(1,4,6,4,0)$. Indeed, there exists the lex ideal $I_{3}$ of $E$ such that $H s_{E / I_{3}}=H_{3}$. It is $I_{3}=\left(e_{1} e_{2} e_{3} e_{4}\right)$.

Using the same notations as in Theorem 3.2 , let $\bar{H}_{3}=H-\widetilde{H}_{3}=(3,12,15,4,0)-$ $(1,4,6,4,0)=(2,8,9,0,0)$. The largest Hilbert sequence that can be extracted from 
$\bar{H}_{3}$ is $H_{2}=(1,4,6,0,0)$. In fact, $H_{2}=H s_{E / I_{2}}$, with $I_{2}=\left(e_{1} e_{2} e_{3}, e_{1} e_{3} e_{4}, e_{1} e_{3} e_{4}, e_{2} e_{3} e_{4}\right)$ lex ideal of $E$.

Next, consider the sequence $\bar{H}_{2}=\bar{H}_{3}-\widetilde{H}_{2}=(2,8,9,0,0)-(1,4,6,0,0)=$ $(1,4,3,0,0)$. The largest Hilbert sequence that can be extracted from $\bar{H}_{2}$ is $H_{1}=$ $\bar{H}_{2}$. The lex ideal whose Hilbert sequence is $H_{1}$ is $I_{1}=\left(e_{1} e_{2}, e_{1} e_{3}, e_{1} e_{4}, e_{2} e_{3} e_{4}\right)$.

We can observe that in such a case the sequence $\bar{H}_{1}=\bar{H}_{2}-\widetilde{H}_{1}=(1,4,3,0,0)-$ $(1,4,3,0,0)=0_{5}$.

Next table describes our procedure:

\begin{tabular}{|c|c|c|c|c|c|}
\hline $\mathrm{H}$-degrees & 0 & 1 & 2 & 3 & \\
\hline$H$ & $(3$, & 12 , & 15 & 4 , & 0 \\
\hline$H s_{E / I_{3}}$ & $(1$, & 4, & 6 , & 4 , & ( \\
\hline$H s_{E / I_{2}}$ & $(1$, & 4, & 6 , & $\mathbf{0}$, & \\
\hline$H s_{E / I_{1}}$ & $(1$, & 4 , & 3 , & $\mathbf{0}$, & \\
\hline $0_{5}$ & $(0$, & 0 , & 0 , & 0 , & \\
\hline
\end{tabular}

Observe that in our situation $f_{1}=f_{2}=f_{3}=0$, and so $\widetilde{H}_{i}=H s_{E / I_{i}}(i=1,2,3)$.

Finally, $M^{\text {lex }}=\oplus_{i}^{r} I_{i} g_{i}$ is the unique lex submodule with Hilbert sequence $H=$ $(3,12,15,4,0)$. More in details:

$M^{\mathrm{lex}}=\left(e_{1} e_{2}, e_{1} e_{3}, e_{1} e_{4}, e_{2} e_{3} e_{4}\right) g_{1} \oplus\left(e_{1} e_{2} e_{3}, e_{1} e_{2} e_{4}, e_{1} e_{3} e_{4}, e_{2} e_{3} e_{4}\right) g_{2} \oplus\left(e_{1} e_{2} e_{3} e_{4}\right) g_{3}$.

Remark 4.2. Note that, given a Hilbert sequence $H$ of a quotient of a free $E$ module $F, \operatorname{rank} F=r$, if one applies to $H r$ repeated subtractions by the nonlargest admissible Hilbert sequences of $K$-algebras $E / T_{i}$, with $T_{i}$ lex ideals of $E$, for $i=1, \ldots, r$ (in the sense of Theorem 3.2 and according to Kruskal-Katona Theorem), then the submodule $N=\oplus_{i}^{r} T_{i} g_{i}$ is not a lex submodule. Otherwise the inclusions in Definition 2.7 are not satisfied, although every $T_{i}$ is lex.

Indeed, let us consider Example 4.1. We can subtract from $H$ the Hilbert sequences $(1,4,6,3,0),(1,4,5,1,0),(1,4,4,0,0)$, and, consequently, we can get the corresponding lex ideals $T_{3}=\left(e_{1} e_{2} e_{3}\right), T_{2}=\left(e_{1} e_{2}, e_{1} e_{3} e_{4}\right), T_{1}=\left(e_{1} e_{2}, e_{1} e_{3}, e_{2} e_{3} e_{4}\right)$. But, $\left(e_{1}, e_{2}, e_{3}, e_{4}\right)^{\operatorname{indeg} T_{2}}=\left(e_{1}, e_{2}, e_{3}, e_{4}\right)^{2} \nsubseteq T_{1}$ and $N=\oplus_{i}^{r} T_{i} g_{i}$ is not a lex submodule.

Example 4.3. Let $E=K\left\langle e_{1}, e_{2}, e_{3}, e_{4}\right\rangle$ and $F=\oplus_{i=1}^{3} E g_{i}$ with $f_{1}=-2, f_{2}=$ $0, f_{3}=2$. Consider the monomial submodule

$$
M=\left(e_{1} e_{2}, e_{3} e_{4}\right) g_{1} \oplus\left(e_{1} e_{2}, e_{2} e_{3} e_{4}\right) g_{2} \oplus\left(e_{2} e_{3} e_{4}\right) g_{3}
$$

of $F$. The Hilbert sequence of $F / M$ is

$$
H s_{F / M}=(1,4,5,4,6,5,6,3,0) .
$$


Setting $J_{1}=\left(e_{1} e_{2}, e_{3} e_{4}\right), J_{2}=\left(e_{1} e_{2}, e_{2} e_{3} e_{4}\right)$ and $J_{3}=\left(e_{2} e_{3} e_{4}\right)$, one has

$$
H s_{E / J_{1}}=(1,4,4,0,0), H s_{E / J_{2}}=(1,4,5,1,0), H s_{E / J_{3}}=(1,4,6,3,0)
$$

and $H_{F / M}(d)=\sum_{i=1}^{3} H_{E / J_{i}}\left(d-f_{i}\right), d \geq-2$, as shown in next table,

\begin{tabular}{|c|c|c|c|c|c|c|c|c|c|c|}
\hline $\mathrm{Hs}$-degrees & -2 & -1 & 0 & 1 & 2 & 3 & 4 & 5 & 6 & \\
\hline$\widetilde{H}_{1}$ & & 4, & 4, & 0 , & 0 & 0 & 0 , & 0 , & 0) & + \\
\hline$\widetilde{H}_{2}$ & $(0$, & 0 & 1, & 4, & 5, & 1 , & 0 , & 0 , & 0) & + \\
\hline$\widetilde{H}_{3}$ & $(0$, & 0, & 0, & 0, & 1, & 4, & 6 , & 3 , & 0) & $=$ \\
\hline$H s_{F / M}$ & $(1$, & 4, & 5, & 4, & 6 , & 5 , & 6 , & , & $0)$ & \\
\hline
\end{tabular}

where $\widetilde{H}_{i}=0_{f_{i}-f_{1}} \uplus H s_{E / J_{i}} \uplus 0_{f_{r}-f_{i}}(i=1,2,3)$. We have indicated the Hilbert sequences $H s_{E / J_{i}}(i=1,2,3)$ in bold.

Let us consider the $[-2,6]$-sequence $H=(1,4,5,4,6,5,6,3,0)$. The largest Hilbert sequence of a graded ideal $I$ that can be extracted from the subsequence $(H(2), \ldots, H(6))=(6,5,6,3,0)$ is $H_{3}=(1,4,6,3,0)$. Indeed, there exists the lex ideal $I_{3}=\left(e_{1} e_{2} e_{3}\right)$ of $E$ such that $H s_{E / I_{3}}=H_{3}$.

With the same notations as in Theorem 3.2. Set $\bar{H}_{3}=H-\widetilde{H}_{3}=(1,4,5,4,6,5,6,3,0)-$ $(0,0,0,0,1,4,6,3,0)=(1,4,5,4,5,1,0,0,0)$, the largest extractable Hilbert sequence from the subsequence $\left(\bar{H}_{3}(0), \ldots, \bar{H}_{3}(4)\right)=(5,4,5,1,0)$ is $H_{2}=(1,4,5,1,0)=$ $H s_{E / I_{2}}$, with $I_{2}=\left(e_{1} e_{2}, e_{1} e_{3} e_{4}\right)$.

Next, consider the sequence $\bar{H}_{2}=\bar{H}_{3}-\widetilde{H}_{2}=(1,4,5,4,5,1,0,0,0)-(0,0,1,4,5,1,0,0,0)$ $=(1,4,4,0,0,0,0,0,0)$. The largest Hilbert sequence that can be extracted from the subsequence $\left(\bar{H}_{2}(-2), \ldots, \bar{H}_{2}(2)\right)=(1,4,4,0,0)$ is $H_{1}=(1,4,4,0,0)=H s_{E / I_{1}}$, with $I_{1}=\left(e_{1} e_{2}, e_{1} e_{3}, e_{2} e_{3} e_{4}\right)$.

We can observe that in such a case the sequence $\bar{H}_{1}=\bar{H}_{2}-\widetilde{H}_{1}=(1,4,4,0,0,0,0,0,0)-$ $(1,4,4,0,0,0,0,0,0)=0_{9}$.

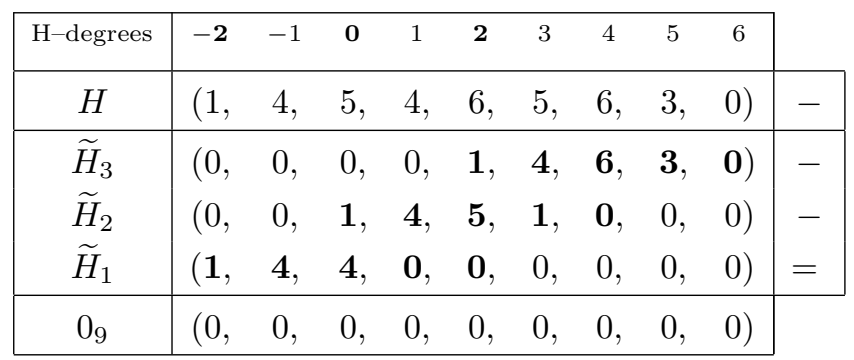

$\widetilde{H}_{i}=0_{f_{i}-f_{1}} \uplus H s_{E / I_{i}} \uplus 0_{f_{r}-f_{i}}(i=1,2,3)$, and we have indicated the Hilbert sequences $H s_{E / I_{i}}(i=1,2,3)$ in bold. Finally,

$$
M^{\text {lex }}=\oplus_{i}^{r} I_{i} g_{i}=\left(e_{1} e_{2}, e_{1} e_{3}, e_{2} e_{3} e_{4}\right) g_{1} \oplus\left(e_{1} e_{2}, e_{1} e_{3} e_{4}\right) g_{2} \oplus\left(e_{1} e_{2} e_{3}\right) g_{3} .
$$


The Hilbert sequence of a quotient of the form $F / M$ can have zeros as initial values. The number of such zeros is $f_{k}-f_{1}$, where $f_{k}$ is the initial critical degree of the Hilbert sequence, as next example will show. Moreover, we can note that the existence of initial zeros implies the presence of some improper ideals as initial components in the direct decomposition. The converse is not true.

Example 4.4. Let $E=K\left\langle e_{1}, e_{2}, e_{3}, e_{4}\right\rangle$ and $F=\oplus_{i=1}^{8} E g_{i}$ with $f_{1}=-3, f_{2}=$ $f_{3}=-1, f_{4}=f_{5}=f_{6}=2, f_{7}=f_{8}=7$. Let us consider the $[-3,7]$-sequence

$$
H=(0,0,1,4,6,7,13,7,1,0,1,4,5,2,0) .
$$

By applying the algorithm in Theorem 3.2, as in the previous examples, we obtain by repeated subtractions from $H$, the following Hilbert sequences $H_{i}=H s_{E / I_{i}}$, with $I_{i}$ lex ideal of $E(i=1, \ldots, 8)$ :

$$
\begin{gathered}
H_{8}=(1,4,5,2,0), H_{7}=(0,0,0,0,0), H_{6}=(1,4,6,1,0), H_{5}=(1,4,1,0,0), \\
H_{4}=(1,4,0,0,0), H_{3}=(1,4,6,4,1), H_{2}=(0,0,0,0,0), H_{1}=(0,0,0,0,0)
\end{gathered}
$$

and

$I_{8}=\left(e_{1} e_{2}\right), I_{7}=E, I_{6}=\left(e_{1} e_{2} e_{3}, e_{1} e_{2} e_{4}, e_{1} e_{3} e_{4}\right), I_{5}=\left(e_{1} e_{2}, e_{1} e_{3}, e_{1} e_{4}, e_{2} e_{3}, e_{2} e_{4}\right)$, $I_{4}=\left(e_{1} e_{2}, e_{1} e_{3}, e_{1} e_{4}, e_{2} e_{3}, e_{2} e_{4}, e_{3} e_{4}\right), I_{3}=(0), I_{2}=E, I_{1}=E$.

\begin{tabular}{|c|ccccccccccccccc|c|}
\hline H-degrees & $-\mathbf{3}$ & -2 & $\mathbf{- 1}$ & 0 & 1 & $\mathbf{2}$ & 3 & 4 & 5 & 6 & $\mathbf{7}$ & 8 & 9 & 10 & 11 & \\
\hline$H$ & $(0$, & 0, & 1, & 4, & 6, & 7, & 13, & 7, & 1, & 0, & 1, & 4, & 5, & 2, & $0)$ & - \\
\hline$\widetilde{H}_{8}$ & $(0$, & 0, & 0, & 0, & 0, & 0, & 0, & 0, & 0, & 0, & $\mathbf{1}$ & $\mathbf{4}$ & $\mathbf{5}$, & $\mathbf{2}$ & $\mathbf{0})$ & - \\
$\widetilde{H}_{7}$ & $(0$, & 0, & 0, & 0, & 0, & 0, & 0, & 0, & 0, & 0, & $\mathbf{0}$ & $\mathbf{0}$, & $\mathbf{0}$, & $\mathbf{0}$, & $\mathbf{0})$ & - \\
$\widetilde{H}_{6}$ & $(0$, & 0, & 0, & 0, & 0, & $\mathbf{1}$ & $\mathbf{4}$ & $\mathbf{6}$, & $\mathbf{1}$, & $\mathbf{0}$, & 0, & 0, & 0, & 0, & $0)$ & - \\
$\widetilde{H}_{5}$ & $(0$, & 0, & 0, & 0, & 0, & $\mathbf{1}$ & $\mathbf{4}$ & $\mathbf{1}$, & $\mathbf{0}$ & $\mathbf{0}$, & 0, & 0, & 0, & 0, & $0)$ & - \\
$\widetilde{H}_{4}$ & $(0$, & 0, & 0, & 0, & 0, & $\mathbf{1}$ & $\mathbf{4}$ & $\mathbf{0}$ & $\mathbf{0}$, & $\mathbf{0}$, & 0, & 0, & 0, & 0, & $0)$ & - \\
$\widetilde{H}_{3}$ & $(0$, & 0, & $\mathbf{1}$ & $\mathbf{4}$ & $\mathbf{6}$, & $\mathbf{4}$ & $\mathbf{1}$ & 0, & 0, & 0, & 0, & 0, & 0, & 0, & $0)$ & - \\
$\widetilde{H}_{2}$ & $(0$, & 0, & $\mathbf{0}$ & $\mathbf{0}$ & $\mathbf{0}$, & $\mathbf{0}$ & $\mathbf{0}$ & 0, & 0, & 0, & 0, & 0, & 0, & 0, & $0)$ & - \\
$\widetilde{H}_{1}$ & $(\mathbf{0}$ & $\mathbf{0}$ & $\mathbf{0}$ & $\mathbf{0}$ & $\mathbf{0}$, & 0, & 0, & 0, & 0, & 0, & 0, & 0, & 0, & 0, & $0)$ & $=$ \\
\hline $0_{15}$ & $(0$, & 0, & 0, & 0, & 0, & 0, & 0, & 0, & 0, & 0, & 0, & 0, & 0, & 0, & $0)$ & \\
\hline
\end{tabular}

$\left(\widetilde{H}_{i}=0_{f_{i}-f_{1}} \uplus H s_{E / I_{i}} \uplus 0_{f_{r}-f_{i}}(i=1, \ldots, 8)\right.$, and the Hilbert sequences $H s_{E / I_{i}}$ $(i=1, \ldots, 8)$ are indicated in bold).

By means of repeated subtractions, we obtain the null sequence $0_{15}$. Hence, the sequence $H$ is the Hilbert sequence of a quotient of a free $E$-module. Indeed, there 
exists the lex submodule

$$
\begin{aligned}
& L=\oplus_{i}^{r} I_{i} g_{i}=E g_{1} \oplus E g_{2} \oplus(0) g_{3} \oplus\left(e_{1} e_{2}, e_{1} e_{3}, e_{1} e_{4}, e_{2} e_{3}, e_{2} e_{4}, e_{3} e_{4}\right) g_{4} \oplus \\
& \quad\left(e_{1} e_{2}, e_{1} e_{3}, e_{1} e_{4}, e_{2} e_{3}, e_{2} e_{4}\right) g_{5} \oplus\left(e_{1} e_{2} e_{3}, e_{1} e_{2} e_{4}, e_{1} e_{3} e_{4}\right) g_{6} \oplus E g_{7} \oplus\left(e_{1} e_{2}\right) g_{8} .
\end{aligned}
$$

such that $H=H s_{F / L}=(0,0,1,4,6,7,13,7,1,0,1,4,5,2,0)=\sum_{i=1}^{8} \widetilde{H}_{i}$.

We close this Section with an example of a sequence of nonnegative integers $H$ that is not a Hilbert sequence of a quotient of a free $E$-modules.

Example 4.5. Let $E=K\left\langle e_{1}, e_{2}, e_{3}, e_{4}\right\rangle$ and $F=\oplus_{i=1}^{3} E g_{i}$ with $f_{1}=-3, f_{2}=$ $-1, f_{3}=2$. Let us consider the $[-3,2]$-sequence

$$
H=(1,2,2,4,3,3,4,5,2,0) \text {. }
$$

By using the Lex-Algorithm and by repeated subtractions from $H$, we obtain the Hilbert sequences $H_{i}=H s_{E / I_{i}}$, with $I_{i}$ lex ideal of $E(i=1,2,3)$ :

$$
\begin{gathered}
H_{3}=(1,4,5,2,0), H_{2}=(1,4,3,1,0), H_{1}=(1,2,1,0,0), \\
I_{3}=\left(e_{1} e_{2}\right), I_{2}=\left(e_{1} e_{2}, e_{1} e_{3}, e_{1} e_{4}\right), I_{1}=\left(e_{1}, e_{2}\right) .
\end{gathered}
$$

Next table describes the construction.

\begin{tabular}{|c|cccccccccc|c|}
\hline H-degrees & $\mathbf{- 3}$ & -2 & $\mathbf{- 1}$ & 0 & 1 & $\mathbf{2}$ & 3 & 4 & 5 & 6 & \\
\hline$H$ & $(1$, & 2, & 2, & 4, & 3, & 3, & 4, & 5, & 2, & $0)$ & - \\
\hline$\widetilde{H}_{3}$ & $(0$, & 0, & 0, & 0, & 0, & $\mathbf{1}$ & $\mathbf{4}$, & $\mathbf{5}$ & $\mathbf{2}$, & $\mathbf{0})$ & - \\
$\widetilde{H}_{2}$ & $(0$, & 0, & $\mathbf{1}$ & $\mathbf{4}$ & $\mathbf{3}$ & $\mathbf{1}$ & $\mathbf{0}$ & 0, & 0, & $0)$ & - \\
$\widetilde{H}_{1}$ & $(\mathbf{1}$, & $\mathbf{2}$ & $\mathbf{1}$ & $\mathbf{0}$ & $\mathbf{0}$ & 0, & 0, & 0, & 0, & $0)$ & $=$ \\
\hline & $(0$, & 0, & 0, & 0, & 0, & $\mathbf{1}$ & 0, & 0, & 0, & $0)$ & \\
& & & & & & & & & &
\end{tabular}

At the end, we do not obtain the null sequence $0_{10}$, and so $H$ is not a Hilbert sequence of a quotient of the given free $E$-module $F$ according to Criterion 3.3.

On the other hand, it is relevant to analyze the second difference that comes into play (according to the Lex-Algorithm):

$\bar{H}_{2}=\bar{H}_{3}-\widetilde{H}_{2}=(1,2,2,4,3,2,0,0,0,0)-(0,0,1,4,3,1,0,0,0,0)=(1,2,1,0,0,1,0,0,0,0)$.

In this case, the largest Hilbert sequence of a graded $K$-algebra $E / I$ is $(1,4,3,1,0)$. In fact, for the sequence $(1,4,3,2,0)$ no ideal $I$ of $E$ with $H s_{E / I}=(1,4,3,2,0)$ does exist (see Kruskal-Katona's theorem). Finally, the submodule

$$
N=\oplus_{i=1}^{3} I_{i} g_{i}=\left(e_{1}, e_{2}\right) g_{1} \oplus\left(e_{1} e_{2}, e_{1} e_{3}, e_{1} e_{4}\right) g_{2} \oplus\left(e_{1} e_{2}\right) g_{3}
$$

has Hilbert sequence $H s_{F / N}=(1,2,2,4,3,2,4,5,2,0)<H . H s_{F / N}$ is the largest extractable Hilbert sequence from $H$ attaining a submodule of $F$. 
Remark 4.6. The procedures described in this paper are part of two Macaulay2 packages "ExteriorIdeals.m2" [2], "ExteriorModules.m2", and tested with Macaulay 1.10. We believe that these packages may reveal useful for further applications. Indeed, functions for computing monomial ideals in a polynomial ring are available in many computer algebra systems, CAS, (for instance, CoCoA [1], Macaulay2 [10] and Singular [8]); on the contrary, to the best of our knowledge, specific packages for manipulating classes of monomial ideals (or monomial submodules) in an exterior algebra have not been implemented yet.

All the examples in this paper have been constructed by such packages.

\section{References}

[1] J. Abbott, A. M. Bigatti and G. Lagorio, CoCoA-5: a system for doing Computations in Commutative Algebra. Available at http://cocoa.dima.unige.it.

[2] L. Amata and M. Crupi, ExteriorIdeals: A package for computing monomial ideals in an exterior algebra, J. Softw. Algebra Geom., 8(1) (2018), 71-79.

[3] L. Amata and M. Crupi, Bounds for the Betti numbers of graded modules with given Hilbert function in an exterior algebra via lexicographic modules, Bull. Math. Soc. Sci. Math. Roumanie (N.S.), Tome 61(109) No. 3 (2018), 237-253.

[4] L. Amata and M. Crupi, A generalization of Kruskal-Katona's Theorem, An. Stiint. Univ. "Ovidius" Constanta Ser. Mat., to appear.

[5] A. Aramova, J. Herzog and T. Hibi, Gotzmann theorems for exterior algebras and combinatorics, J. Algebra, 191(1) (1997), 174-211.

[6] W. Bruns and J. Herzog, Cohen-Macaulay Rings, Cambridge Studies in Advanced Mathematics, 39, Cambridge University Press, Cambridge, 1993.

[7] M. Crupi and C. Ferrò, Squarefree monomial modules and extremal Betti numbers, Algebra Colloq., 23 (3) (2016), 519-530.

[8] W. Decker, G. M. Greuel, G. Pfister and H. Schönemann, Singular 4-1-0 A computer algebra system for polynomial computations, (2016), available at http://www. singular.uni-kl.de.

[9] D. Eisenbud, Commutative Algebra, With a view toward algebraic geometry, Graduate Texts in Mathematics, 150, Springer-Verlag, New York, 1995.

[10] D. R. Grayson and M. E. Stillman, Macaulay2, a software system for research in algebraic geometry, available at http://www.math.uiuc.edu/Macaulay2.

[11] J. Herzog and T. Hibi, Monomial Ideals, Graduate texts in Mathematics, 260, Springer-Verlag, London, 2011.

[12] G. Katona, A theorem of finite sets, in Theory of graphs (Proc. Colloq., Tihany, 1966), Academic Press, New York, (1968), 187-207. 
[13] J. B. Kruskal, The number of simplices in a complex, in Mathematical optimization techniques (R. Bellman, ed.), University of California Press, Berkeley, (1963), 251-278.

[14] F. S. Macaulay, Some properties of enumeration in the theory of modular systems, Proc. London Math. Soc., 26 (1927), 531-555.

[15] R. P. Stanley, Cohen-Macaulay rings and constructible polytopes, Bull. Amer. Math. Soc., 81 (1975), 133-135.

Luca Amata and Marilena Crupi (Corresponding Author)

Department of Mathematics and Computer Sciences

Physics and Earth Sciences

University of Messina

Viale Ferdinando Stagno d'Alcontres 31

98166 Messina, Italy

e-mails: lamata@unime.it (L. Amata)

mcrupi@unime.it (M. Crupi) 